\title{
Enhanced integration of flow-based market coupling in short-term adequacy assessment
}

\author{
Bashir Bakhshideh Zad ${ }^{1 *}$, Jean-François Toubeau ${ }^{1}$, Behzad Vatandoust ${ }^{1}$, \\ Kenneth Bruninx ${ }^{2,3}$, Zacharie De Grève ${ }^{1}$, François Vallée ${ }^{1}$ \\ ${ }^{1}$ Power Systems and Markets Research Group, University of Mons, Belgium \\ ${ }^{2}$ Energy Systems Integration and Modeling group, University of Leuven, Belgium \\ ${ }^{3}$ EnergyVille, Belgium \\ *Corresponding author, email address: bashir.bakhshidehzad@umons.ac.be
}

\begin{abstract}
The resource adequacy of the interconnected Central Western Europe (CWE) electricity system is assessed considering the cross-border exchange capacities defined through the Flow-Based (FB) domains. Integration of FB domains into adequacy assessments poses several challenges since the FB domains depend on factors which are not known over the horizon of adequacy study. Computing hourly FB domains for each generated scenario of adequacy study, firstly, requires adopting assumptions on those unknown parameters (that may not fully match with the reality). Secondly, it noticeably increases the computational complexity of the study. The above challenges can, however, be circumvented by the datadriven alternatives. This paper presents a novel clustering technique for FB domains, which is specifically tailored for adequacy assessments. In contrast to the classical approach employed by the CWE Transmission System Operators (TSOs), which clusters the FB domains based on their overall geometrical resemblance, the proposed technique relies on the maximum and minimum zonal balances allowed by the FB domains, which are decisive factors in the CWE resource adequacy assessments. Indeed, during scarcity moments, the zonal net positions (balances) tend to reach their extreme values to reduce the costs of energy not served. The proposed goal-oriented clustering technique is examined against the classical clustering methodology employed by the CWE TSOs. The conducted simulations demonstrate that the proposed technique considerably (by a factor of over 5.5) improves the accuracy of the CWE adequacy assessments while being scalable with the future evolution of the Flow-based Market Coupling (FBMC). As such, it has direct implications for the adequacy assessment considering the FB domains.
\end{abstract}

Keywords - adequacy assessments, flow-based market coupling, clustering analysis, crossborder energy exchanges.

\section{INTRODUCTION}

Adequacy assessments evaluate ability of an electric power system to meet the load demand over the studied (future) horizon under various working conditions and different contingencies. Traditionally, the adequacy assessment has been carried out according to a deterministic formulation based on the amounts of peak load demands and available generations under conservative contingency assumptions. The growing integration of 
renewable-based generations into the power network has introduced new uncertainties in the electric power system [1], [2]. In addition, the liberalization of the electricity sector requires a cost-effective planning and operation of power network, which cannot be achieved through deterministic-based calculations [3], [4]. In order to efficiently cope with the increasing uncertainty while addressing the cost-effectiveness, the adequacy assessment methodologies have been changed from deterministic towards risk-based approaches [5]. In the latter category, Monte Carlo simulations are usually carried out to capture the uncertain nature of load and generation as well as the unplanned outages. Sequential and non-sequential Monte Carlo methods for generation adequacy assessment in single- and multi-area electric power systems have been studied in [6]. Also, Monte Carlo simulations have been utilized in [7], [8] to integrate wind generation into adequacy assessments. An importance-based Monte Carlo sampling approach has been developed in [9] that improves the computational performance of the considered adequacy problem. Alternatively, non-sampling-based methodologies relying on analytical approaches [10], [11], data-driven techniques [12] and neural networks [13] have been also investigated in the literature.

Besides the available domestic generation and load demand, another important factor in the adequacy study of an interconnected electric power system is the cross-border exchange capacities between control areas (i.e., the amount of achievable import or export via the interconnections) [14]. Currently, two approaches are being used for incorporating the crossborder exchanges into the electricity market in Europe, introduced as follows. The Net Transfer Capacity (NTC) that assumes a commercial capacity between two market zones, and the Flow-Based (FB) approach, which is designed to (more) accurately consider the physical grid constraints. The NTC is the traditional capacity allocation approach still being used on specific borders in Europe, while the Central Western Europe (CWE) region, which is the focus of the current paper, has moved towards the Flow-Based Market Coupling (FBMC) since 2015. The FBMC is the target model to be applied to other regions of Europe [15]. Currently, there are 5 zones (hubs) in the FBMC. The FB domains are thus 5-dimensional (5D) polytopes where vertices of FB polytopes define the possible exchanges of each zone with the CWE electricity system.

In order to incorporate the interconnection capacities through the FB domains into the riskbased adequacy assessments, the main challenge consists in finding the FB domain that correctly corresponds to each generated scenario. Indeed, the FB domains depend on factors such as network operating points and exogenous conditions (e.g., meteorological circumstances affecting the load demands and renewable generations) [16], which cannot be precisely known over studied horizon of the adequacy assessment. In addition, the internal parameters of FBMC such as the generation shift keys and the considered minimum threshold can have high impacts on FB domains and the market outcomes as studied in [17], [18], [19], [20]. In such a context, computation of hourly FB domain for each scenario of risk-based adequacy study, firstly, requires adopting assumptions on those parameters (that may not correctly match with the reality), secondly, it noticeably increases the computational complexity of the adequacy assessment.

The abovementioned challenges can, however, be avoided by moving to pure data-driven approaches. In this regard, the French TSO (RTE), Belgian TSO (Elia) and European TSOs (ENTSO-E) employ a two-step methodology, which consists of first clustering the historical FB domains, then correlating the obtained FB clusters with relevant factors [21], [22], [23], 
[24]. The main objectives of clustering task are to group the historical FB domains into a reduced number of clusters, and to select (compute) the representative object (prototype) of each cluster. Focusing on the clustering phase of the above methodology, the authors in [25] propose solutions to improve the performance of the clustering procedure employed by the TSOs. In this regard, new distance measures based on the volume calculation of FB domain (polytopes) and the Hausdorff distances are proposed, and the performance of various clustering algorithms are tested. The internal validation techniques are then utilized to evaluate the quality of cluster results. It is demonstrated that the fuzzy clustering approach can improve the FB domain cluster results evaluated by the Silhouette index. It is also shown that the classical k-medoids approaches are sensitive to initialization step and that the recent variants of k-medoids approach can lead to more robust and improved results.

Despite the differences of studied distance measures in [25] with the one used by the TSOs, they have all one feature in common that is to evaluate the dissimilarity between two FB domains based on their overall shapes (i.e. shape-based measures). The main drawback of such distance measures is that it gives the same degree of importance to all the vertices of FB domains while the focus of the adequacy study is on the scarcity conditions, where the maximum feasible exchanges (defined by extreme zonal net positions) would take place to reduce the need for load shedding. To make it more vivid, Fig. 1 presents the vertices of a typical 5D FB domain projected in a two-dimensional (2D) plane. The black stars in the figure show the ordinary vertices of FB domain polytope while the red points illustrate the vertices representing maximum possible exchanges of each zone (country). As stated earlier, during the scarcity period, the tendency of exchanges is towards (one of) the red points; consequently, the cross-border exchanges will not be limited to other vertices located for instance in the center of the graph. In other words, the vertices shown with black stars do not have the same degree of importance as the ones representing the maximum exchanges illustrated with the red points, in the context of adequacy assessments.

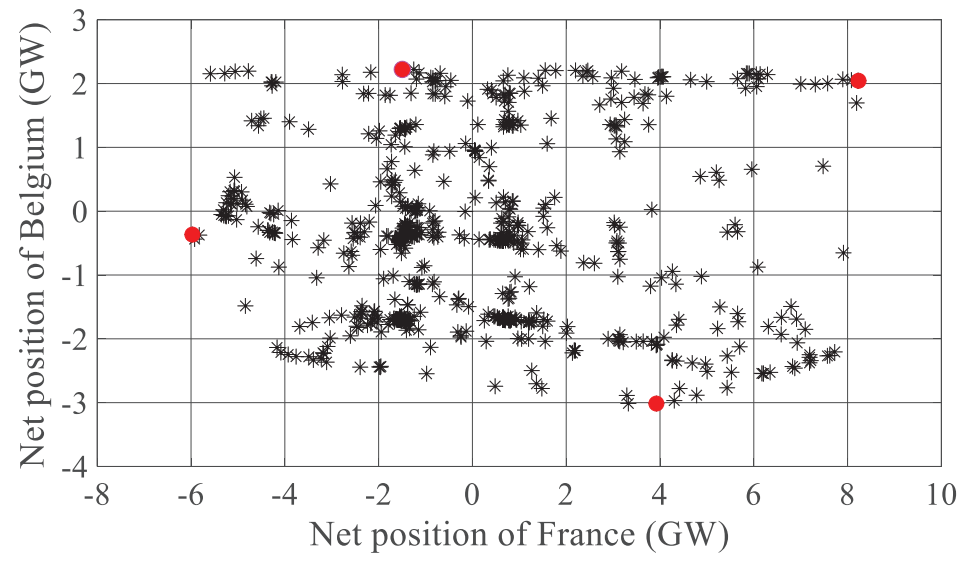

Figure 1: Vertices of a typical 5D FB polytope projected in a 2D plane

The current paper presents a novel clustering technique, according to which, the FB domains are grouped based on their extrema zonal (per each zone) net positions. This choice is motivated by the fact that at the time of scarcity, the zonal exchanges tend to reach their extrema capacities in order to minimize the costs of the energy not supplied, which are considerably higher than the generation costs. This phenomenon can be explained by the 
market coupling mechanism and from an optimization perspective. In such an environment, in order to achieve the price convergence in different countries (zones), the interconnections are exploited as much as required. Consequently, during scarcity period, market will be cleared in one of the extreme vertices of FB domain (to reduce the zonal price discrepancies).

In view of the above discussion, the main contribution of this paper is to develop a clustering technique for FB domains that:

- Enhances the accuracy of FB domain assignment task within the adequacy assessments of CWE electricity system.

- Covers the potential scalability issue of the clustering approach being used by the TSOs when new countries will join to the FBMC.

- Lessens the computational burden of probabilistic adequacy assessments in the CWE electricity system.

The proposed goal-oriented clustering technique; therefore, can have direct contribution to enhancement of the accuracy, scalability, and computational tractability of the adequacy assessments in the CWE electricity system, as explained below.

Improved accuracy: the proposed goal-oriented technique gathers the FB domains that have similar extrema points in the same cluster. This enables us to define the cluster prototypes, which can correctly represent their respective FB domains (placed in the same group) in the context of adequacy studies. Consequently, it can improve the accuracy of the FB domain assignment task in the adequacy study. The proposed technique covers the drawback of the methodology employed by the CWE TSOs that clusters the FB domains based on their overall geometrical shapes. The FB domains are polytopes consisting of several hundred (up to 1000 and more) vertices. In order to calculate the dissimilarity between overall shapes of two FB domains, the final distance (dissimilarity) between two FB domains is calculated by aggregating all individual distances between each pair of their vertices (see (2)). Doing so, the final distance becomes too general such that different FB domains having various shapes can lead to a similar value as their differences can be covered in the final distance. Therefore, clustering the FB domains based on their overall shapes can lead to an erroneous identification of the FB domain prototype (to be used in adequacy assessments).

Scalability: the number of vertices of a FB domain polytope is highly sensitive to the number of its dimensions, and it drastically increases as a function of the latter. This implies that when new countries join to the FBMC, the number of vertices of FB domain polytope can reach several thousands. It is expected that the distance measure employed by the CWE TSOs would not be able to effectively compute the FB domain dissimilarities within this new situation, due to its aggregation feature explained above. In contrast, the proposed goaloriented clustering technique only considers the extrema zonal (per each zone) net positions of each FB domain according to (3). Consequently, when a new country joins to the FBMC, only two more vertices (representing extrema exchanges of that country) needed to be taken into account. This feature permits the proposed clustering technique to efficiently handle the curse of dimensionality of the evolving FBMC.

Calculation time: by only focusing on the vertices representing the maximum exchange capacities, the proposed goal-oriented clustering approach considerably reduces the computation burden needed to calculate the dissimilarity between each pair of FB domains in 
studied dataset. Therefore, it presents a suitable approach for dealing with the large FB domain datasets.

The remainder of this paper is structured as follows. Section II introduces the definition of FB domains, expresses the challenge for integrating FB domains in adequacy assessments, and presents the current methodology used by the CWE TSOs to this end. The proposed goaloriented clustering technique is described in Section III and the developed clusteringadequacy framework to examine the performance of studied clustering methods is presented in Section IV. Afterwards, Section V introduces the considered test cases and reports the simulation results, followed by the discussion on the results in Section VI. Finally, the last section is dedicated to the paper conclusions.

\section{ADEQUACY ASSESSMENT INCLUDING FB DOMAINS}

\section{A. Cross-border exchange capacities defined through the Flow-Based (FB) domains}

The FBMC aims at modelling the physically feasible power exchanges for the cross-zonal trades taking into account the interdependencies of flows within the zone (countries). In practice, there is a fundamental difference between commercial exchanges and physical flows, since in a power network, the electric power flows through the existing paths according to the Kirchhoff's laws. Consequently, the exchange capacity between two market zones cannot be fully allocated to the commercial trade between them, as a part of the capacity could be used by flows resulting from the trade of other market zones [26].

In the FBMC, the physical possible power flows within the selected network elements are determined according to the FB domains, which are represented as a set of linear constraints in the following form.

$$
\boldsymbol{A} \times \boldsymbol{x} \leq \boldsymbol{b}
$$

where $\boldsymbol{A}$ is a matrix containing the Power Transfer Distribution Factors (PTDF), $\boldsymbol{x}$ gives the net position (=export-import) of each zone or country, and $\boldsymbol{b}$ is the vector of the Remaining Available Margin (RAM) of the selected grid elements. The PTDF coefficients indicate the incremental physical flows induced on transmission lines as a result of a power exchange between two zones. Each row of the system of linear constraints (1) represents one of the selected grid elements. The FB domain at a given hour corresponds to the intersection of all half-spaces created by the system of linear constraints (1), which will eventually construct a $N$-dimensional polytope (with $N$ denoting the number of zones involved in the FBMC). The vertices of a FB domain polytope define the possible exchanges of each country with other countries in the CWE region. A typical 5D FB domain polytope that represents around 100 selected grid elements can include more than several hundred vertices.

\section{$B$. The main challenge for incorporating the FB domains into the adequacy study}

A FB domain represented through the system of linear constraints (1) is integrated into the adequacy assessments by adding (1) to the optimization problem that is at the core of the adequacy assessments. Integrating the FB domains into the adequacy assessments however poses several challenges. The main difficulty consists in finding, for each generated scenario of the risk-based adequacy assessments, the FB domain that correctly represents the network constraints and exogenous conditions of the studied situation. Indeed, the shape and size of a typical FB domain depend on the above parameters as well as on the internal parameters of 
the FBMC (e.g., generation shift keys), which are not known over the studied horizon of adequacy simulations. The FB domains can take various forms and shapes according to those parameters. In order to present the variability of FB domains, Fig. 2 demonstrates 96 (hourly) FB domains (corresponding to 4 randomly selected days in January 2020), which are projected and plotted in the Germany-France plane. As it can be seen, the FB domains can take various values ranging for instance, for possible import of Germany, from almost $8 \mathrm{GW}$ to $14 \mathrm{GW}$ (negative values indicate the import capacity). Obviously, such a wide range of variations in possible cross-border exchanges can totally change the final adequacy results. It should be noted that this figure does not represent the coordinates related to the 3 other dimensions. In reality, a feasible exchange is determined in accordance with coordinates of all the 5 dimensions. Consequently, an exchange to be feasible must be contained inside the FB domain polytope.

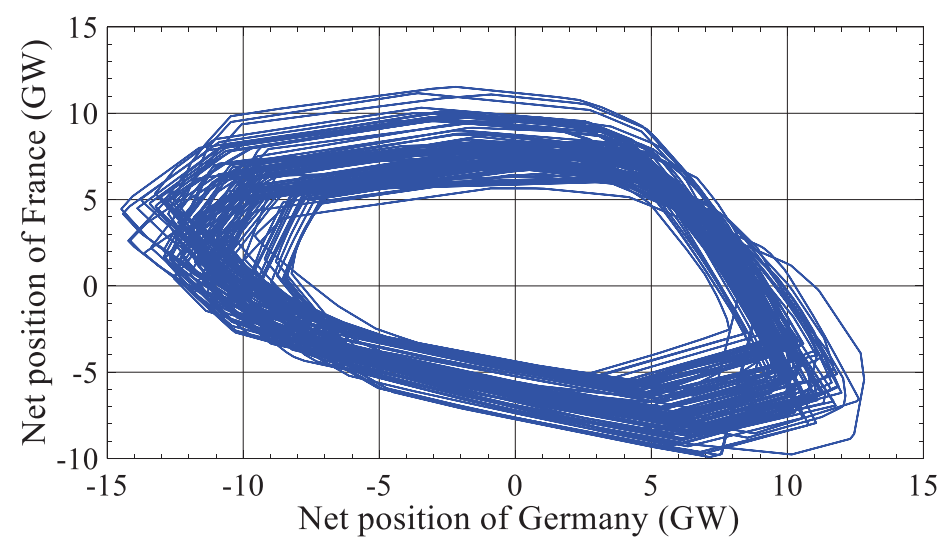

Figure 2: 96 hourly FB domains corresponding to 4 randomly selected days in January 2020 projected in France-Germany plane (data source: [27])

\section{Current methodology employed by the CWE TSOs}

Computation of hourly FB domains is a sequential task that requires information about several factors that are unknown over the studied horizon of the adequacy study and needs data exchange among the involved parties (TSOs). Simulating such a procedure for each scenario of the risk-based adequacy study is a complex task that noticeably increases the computation burden of the study. To address these challenges, the French TSO (RTE), Belgian TSO (Elia) and European TSOs (ENTSO-E) rely on a two-step strategy based on a clusteringcorrelation procedure of historical FB domains [21], [22], [23], [24] which is summarized below.

The objective of the first step is to cluster the historical FB domains into a limited number of groups according to their geometrical similarities. A partitional clustering algorithm is employed to that end, in combination with a dissimilarity (or distance) measure, which compares the geometrical shapes of the FB domains. More precisely, the distance (dissimilarity) between two arbitrary FB domains is computed using the coordinates of their polytope vertices as shown in Fig. 3, in a 2D plane. Let $A$ and $B$ be two sets of points, with $A=\left\{a_{1}, a_{2}, \ldots, a_{n}\right\}$ and $B=\left\{b_{1}, b_{2}, \ldots, b_{m}\right\}$, which represent vertices of two selected FB domain polytopes. The distance from the FB domain $A$ to the FB domain $B$ is calculated as follows. 


$$
d_{T S O}\left(F B_{A}, F B_{B}\right)=\sum_{a \in A} \min _{b \in B} \sqrt{(a-b)^{2}}
$$

The above equation expresses that the Euclidean distance between each vertex of $A$ and the corresponding closest vertex from $B$ is summed to constitute the final distance between the FB domain $A$ and the FB domain $B$. For the sake of clarity, Fig. 3 depicts two FB domains projected and plotted into a 2D plane. The vertices of FB domains are marked with small points. The abovementioned distance from the FB domain $A$ to the FB domain $B$ is equal to the sum of lengths of the red vectors connecting each vertex of the former FB domain to its nearest counterpart from the latter FB domain. Equation (2) is applied to calculate the distance between each pair of FB domains. In the end, a square matrix is constructed that includes all distances (dissimilarities) of the FB domains in the studied dataset.

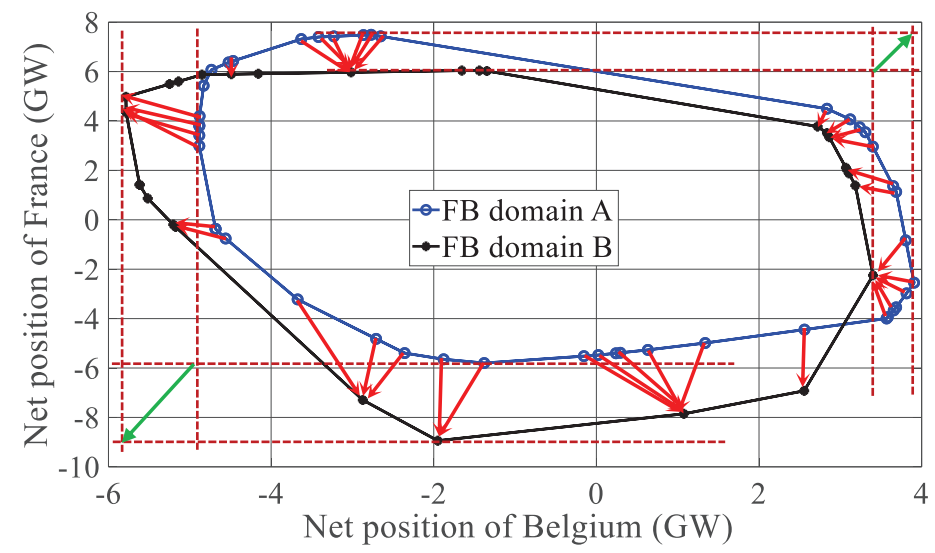

Figure 3: Illustration of studied distance measures that compares the dissimilarity between two randomly selected FB domains in a 2D plane

The $k$-medoids clustering algorithm is then applied to the calculated distance matrix. It consists in a partitional clustering algorithm, which structures the input space by assigning each data object to the cluster with the closest medoid. In the end of the clustering phase, each FB domain is assigned to one specific cluster.

The second stage of the above methodology is the correlation phase, which aims to identify a link between the partitioned FB domains and external factors such as generations and load demands in different countries. Indeed, risk-based adequacy analysis relies on the Monte Carlo sampling of those above factors. Therefore, considering FB domains that are in line with the sampled scenarios is crucial in a risk-based adequacy assessment. The shape of a FB domain is affected by several components of different importance levels. The objective is to carry out the correlation study with the most important ones affecting the FB domains. The FB domain clusters obtained in the previous phase are finally correlated with the selected factors to calculate the probability of occurrence of each FB cluster for each factor combination (e.g., high, medium, and low levels). Depending on the magnitude of the studied factors in each generated Monte Carlo scenario, the medoid of the cluster with the highest probability is employed, and the linear constraints encoded by that medoid are used in the adequacy assessment.

It should be noted that by relying on the historical FB data, the above clustering-correlating methodology is applicable to short-term adequacy assessments. For instance, to evaluate the 
risk of load shedding in the next winter, a common practice, which is annually performed in Belgium by Elia (the Belgian TSO, see [24]). The longer-term adequacy assessments (e.g., having horizon of 5 years or more) must consider the structural changes of electric power system such as adding new interconnections, countries (zones) in the market coupling or phasing out of part of generation share. These structural changes cannot be captured via historical data and are out of scope of the present work.

\section{GOAL-ORIENTED CLUSTERING OF FB DOMAINS}

The goal-oriented clustering of FB domains is proposed in this work. Unlike the clustering technique employed by the CWE TSOs that considers all the vertices of FB domain, the goaloriented clustering focuses on the vertices, which are decisive in the context of adequacy study. During the scarcity periods (i.e., the main interest of adequacy assessment), net position of each country tends to reach its extremum capacity to minimize the cost function of the adequacy study. When one country needs importing, the countries which are not experiencing power shortage will export power to the country in need to reduce (ideally remove) the generation-demand mismatch to minimize the cost of energy not served. Therefore, the maximum import capacity (i.e., the vertex with the minimum balance) of the country in need, and maximum achievable exports from other countries are decisive in the adequacy results. When two (or more) countries simultaneously require importing power, the available generation (for export) will be shared among the countries in need. The maximum possible exports from countries which are not experiencing power shortage are again important parameters while the maximum import capacities of countries in need may not be fully exploited since the available energy should be shared between (among) them.

In the light of the above context, the goal-oriented comparison of FB domains is proposed in this work, which measures the FB domain dissimilarities only with respect to the maximum import and export capacities of each country participating in the FBMC. To this end, we firstly select for each country, its maximum achievable import and export net positions. By convention, a positive net position shows that the country is exporting, and a negative balance means that the country is importing. Therefore, for each dimension (country) of the FB domain polytope, its maximum and minimum net positions are selected. The dissimilarity between two FB domains based on the proposed goal-oriented distance measure is obtained by calculating the Euclidean distance between their maximum zonal net positions as well as their minimum zonal net positions according to:

$$
d_{G O}\left(F B_{A}, F B_{B}\right)=\sqrt{\sum_{i=1}^{N}\left[\left(N P_{A, i}^{\max }-N P_{B, i}^{\max }\right)^{2}+\left(N P_{A, i}^{\min }-N P_{B, i}^{\min }\right)^{2}\right]}
$$

where $N$ is the number of zones involved in the FBMC, $N P_{A, i}^{\max }$ and $N P_{A, i}^{\min }$ denote the maximum and minimum net positions of the FB domain $A$ in zone $i$, respectively. Fig. 3 illustrates the distance between two (previously chosen) FB domains by applying equation (3) to the 2D plane. The above distance is equal to the sum of lengths of two green vectors connecting the extreme points of the FB domains. In Fig. 3, one can observe the difference between the goal-oriented technique and the distance measure currently employed by the CWE TSOs. In the latter, all the vertices shown in 2D plane are considered for comparison of two FB domains. However, in Fig. 3, the shown vertices are obtained by applying the convex hull of all the vertices projected to 2D plane (while neglecting the coordinates related to the 3 
other dimensions). In reality, a typical 5D FB domain consists of several hundred (up to 1000 and more) vertices. When we consider all these vertices for comparison between two FB domains (according to the distance measure used by the TSOs), the final distance will be formed by the aggregation of all the respective individual distances. Consequently, the distance between those extreme vertices (i.e., important in the adequacy study) will be dominated by the sum of distances between the rest of vertices (by a factor of several hundred and more). In addition, the final aggregated distance between two FB domains according to (2) becomes too general such that various polytopes with different shapes can lead to a similar final distance since their differences can be covered in the final value. As a result, when the distance measure used by the CWE TSOs is employed in the clustering process, the FB domains clustered in one group can have different characteristics in the context the of an adequacy evaluation, leading to totally different results.

In contrast to the technique used by the CWE TSOs, the goal-oriented distance measure only considers the maximum and minimum net positions of each country. Thus, the final distances obtained by this method can fully reflect the dissimilarities between the studied FB domains with respect to their maximum and minimum net positions. This will lead to the selection of cluster prototypes, which can correctly represent their respective objects (placed in the same group) in the context of the adequacy study. Given that the maximum and minimum exchanges of each country shape the final adequacy results (since interconnections will be used as much as feasible in the scarcity conditions), we can expect that when the selected cluster prototype according to the goal-oriented technique is employed in the adequacy study, it can lead to the results, which are relatively similar to the ones that could be obtained by using the exact FB domains. In this way, the goal-oriented clustering approach can offer an improved manner of integrating cross-border exchange capacities into the adequacy assessments.

\section{Proposed Clustering-Adequacy Framework For Validation Purposes}

The performance of the proposed goal-oriented clustering technique is examined against the classical methodology employed by the CWE TSOs in a framework of clusteringadequacy study. The procedure for conducting the simulations and analyses of this paper is shown in Fig. 4 and described as follows. The data processing step and the clustering task are implemented in the R environment and the adequacy assessments are performed in Matlab.

\section{A. Processing of historical database}

In the first step, we need to obtain the vertices of historical FB domains (polytopes) in the studied dataset. To do so, the vertex enumeration operation is carried out [28] on the FB domains modelled with a set of linear constraints (see (1)). Then, the studied distance measures, namely the one developed by the CWE TSOs (explained in Section II.C) as well as the novel goal-oriented technique proposed in this paper (Section III) are separately employed to construct the matrix of distances. The latter includes the dissimilarity between each pair of FB domains in the studied dataset.

\section{B. Clustering analysis}

In the second step, a partitional clustering algorithm is applied to the distance matrix calculated in the previous stage in order to group the FB domain data into a predefined number

of clusters. In this work, the clustering task is carried out according to the $k$-medoids algorithm 
employed by the CWE TSOs [15], [22], [23], [24]. The $k$-medoids algorithm structures the input space by assigning each data object to the cluster with the closest representative object (or prototype). With $k$-medoids, the cluster representatives are their respective medoids, i.e., the data objects that minimize the sum of distances with all the objects of the considered cluster. Cluster representatives are therefore existing physical objects with $k$-medoids. The Partitioning Around Medoids (PAM), which is the classical approach of the $k$-medoids family is utilized to conduct the clustering analysis of this paper [29].

Once the clustering process is completed, each FB domain in the dataset will be assigned to one specific cluster, and there will be one representative for each cluster (i.e., the medoid of that cluster). In the adequacy simulations (explained in the next part), the representative FB domains (i.e., the medoids) will replace their respective FB domains placed in the same group.

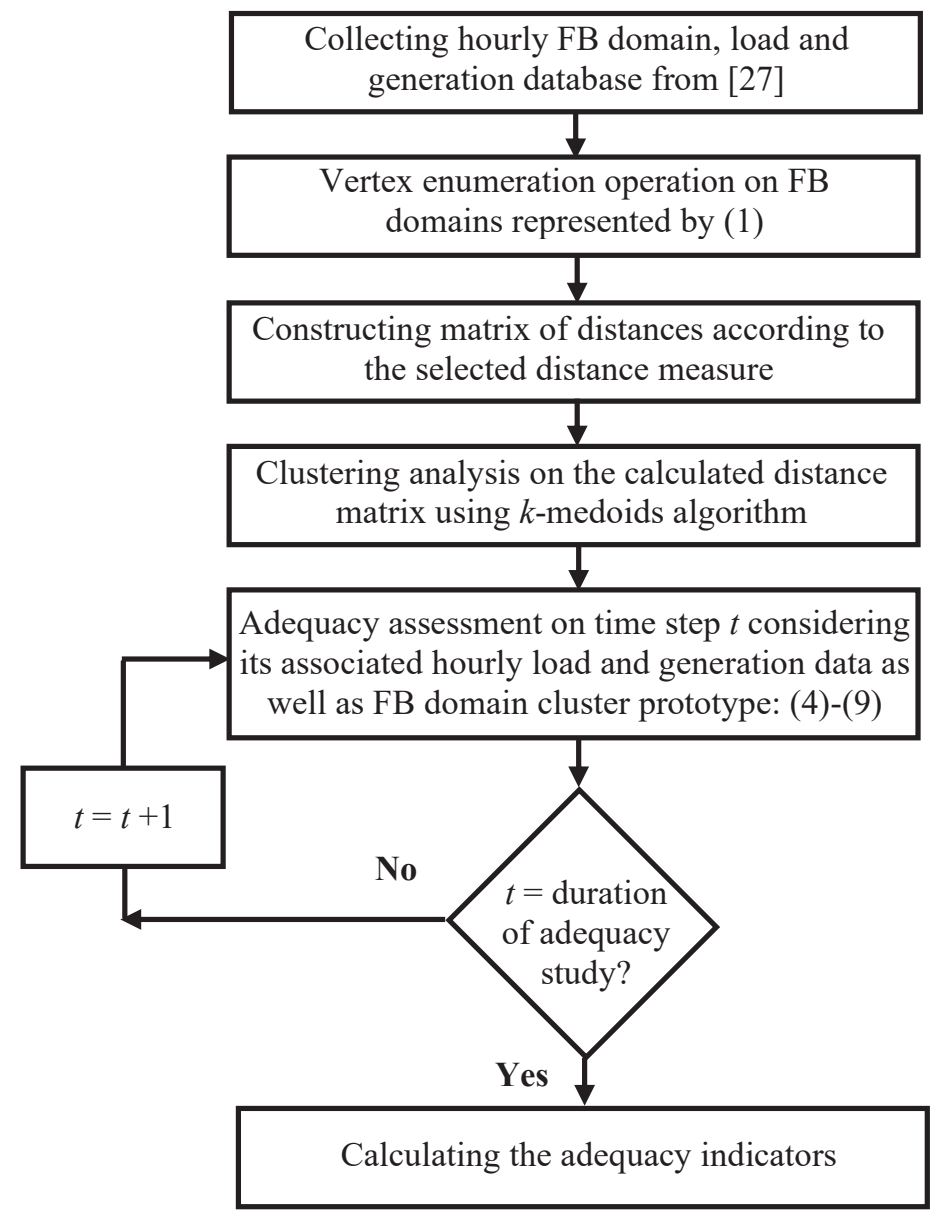

Figure 4: Flowchart summarizing the developed clustering-adequacy framework

\section{Adequacy assessments}

The third step of the proposed framework is dedicated to the adequacy simulations. In this regard, an economic dispatch program customized for the adequacy study of the CWE electricity system considering the FB domains is developed here. Relying on a linear optimization formulation, the economic dispatch tool aims at minimizing the Energy Not 
Served (ENS) while respecting the FB domain as well as the zonal match of load-generation. It is formulated as follows.

$$
\begin{gathered}
\text { Minimize: } \sum_{i=1}^{N} E N S_{i} \\
G_{i}-L D_{i}+N P_{i}+E N S_{i}=0 \quad \forall i \in N \\
\sum_{i=1}^{N} N P_{i}=0 \\
0 \leq G_{i} \leq G_{i}^{\max } \\
0 \leq E N S_{i} \\
\text { PTDF } \times \boldsymbol{N P} \leq \mathbf{R A M}
\end{gathered}
$$

where $N$ is the number of countries (zones) participating in the $\operatorname{FBMC}(N=5), G_{i}$ denotes the aggregated generation of zone $i, L D_{i}$ gives the load demand in zone $i$ and $N P_{i}$ stands for the net position of zone $i$ (=export-import). The decision variables of optimization problem (4)(9) are the zonal ENS $\left(E N S_{i}\right)$ as well as the zonal generations $\left(G_{i}\right)$. The objective function of the optimization problem is stated in (4). Equation (5) reflects the fact that the sum of generation and power exchanges via the interconnections must be equal to the load demand in each zone. In order to ensure the feasibility of problem at each studied time step, the slack variable i.e., $E N S_{i}$ is added to (5) to cover the possible generation shortage of each zone. It will be used only when domestic generation as well as the power exchanges via interconnections cannot cover the zonal load demands. Equation (6) expresses that the sum of zonal net positions in the CWE electricity system must be equal to zero. Constraint (7) considers the upper and lower bounds on the zonal generations and constraint (8) defines that the ENS is limited to non-negative values. Finally, the system of linear constraints (9) incorporates the representative FB domain selected in the clustering phase into the adequacy simulations. The components of (9) are PTDF matrix, vector of zonal net positions (NP) and RAM vector as introduced in Section II.A. Given that the FB domains have been initially modelled with respect to the net positions (NP), a replacement operation is required to convert the NP vector into the decision variables of the above optimization problem. Fig. 5 represents the interconnected CWE electricity system that has been modelled in this work.

The presented optimization problem will be solved for each time step $t$ (hour) of the adequacy study considering its associated hourly available generation and load demand in each zone as well as its corresponding representative FB domain obtained in the clustering phase. In other words, during the adequacy simulations, the hourly historical FB domains are replaced by their respective cluster prototypes and simulations are carried out on the cluster representatives. It should be noted that there is no Monte Carlo sampling procedure in adequacy simulations of this work since the "true" FB domains corresponding to these (artificial) scenarios are not known. Instead, in this paper, relying on real (historical) hourly FB data (see Section V), we aim at improving the performance of the FB clustering task to be used in the adequacy assessments. The (improved) FB domain clusters can be linked to relevant exogenous factors in post-processing via a correlation analysis as employed by the CWE TSOs (see Section II.C). In this way, it is possible to link the FB domain prototypes to the generated Monte Carlo scenarios and integrate them into the risk-based adequacy assessments. 


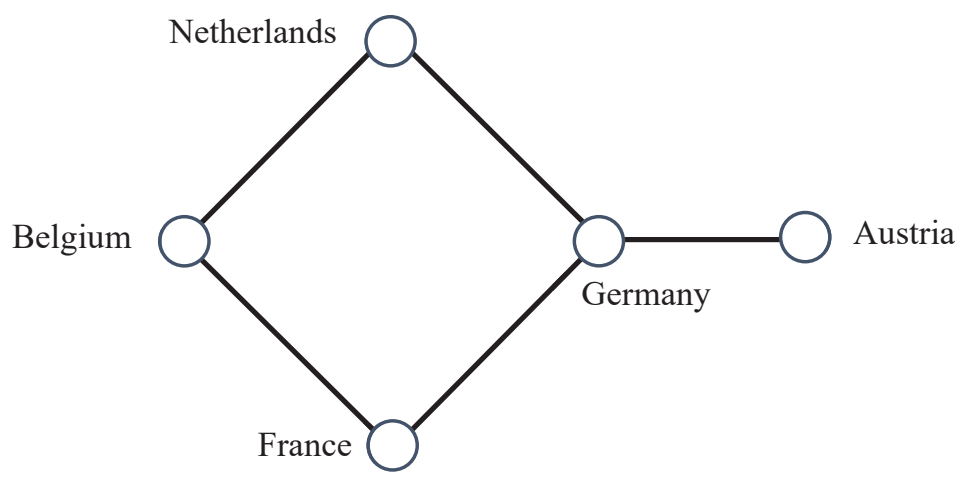

Figure 5: Illustration of the considered CWE electric power system

It is worth noting that the developed economic dispatch model does not consider the energy exchanges of CWE countries with the countries outside this region since these exchanges are not defined in accordance with the FB domains. In addition, given that the focus of this study is on the role (behavior) of interconnections during the scarcity periods, the developed optimization tool does not take into account the (minimization of) generation costs. Indeed, during the scarcity periods, every possible measure would be taken to avoid the load shedding and to reduce the ENS. Therefore, discrepancy of zonal generation costs does not constitute an important factor.

\section{Calculating adequacy indicators}

In the last stage, the adequacy-related indices will be calculated over the considered horizon of the adequacy study. Two adequacy indices will be evaluated for each country (zone) namely the Loss Of Load Probability (LOLP) in \% as well as the Total Energy Not Served (TENS) in GWh $[5,30]$. The TENS is equal to the sum of the value of the slack variable $E N S_{i}$ over the studied horizon of the adequacy study. The LOLP gives the number of activations of the slack variable divided by the number of time steps of the studied horizon.

\section{Clustering-AdeQuacy ANALYSES}

\section{A. Input data}

In line with the current practice of the adequacy assessment in Europe, which evaluates the ability of electric power system to cover the load demand from a day-ahead market perspective [21], [24], we conduct our simulations from the same viewpoint but on the historical data. The clustering-adequacy study presented in Section IV is conducted on 5808 hourly FB domains, which correspond to the period from $23^{\text {rd }}$ January 2020 to $20^{\text {th }}$ September 2020 (i.e., the latest available data at the time of execution of this work) [27]. The five zones in the CWE region participating in the FBMC are modelled in the adequacy assessment according to their total hourly loads and generations over the same period (as chosen for the FB domains). The 2 Days-ahead Congestion Forecast (D2CF) available at [27] is used to this end. It provides the best estimate of the state of the CWE interconnected system for the delivery day. The D2CF data includes the aggregated hourly load demand and available generation in each CWE zone. The remaining available margin (RAM) of the considered FB domains has been also calculated according to the D2CF data [15]. 


\section{B. Test cases}

The performance of the distance measure developed by the CWE TSOs (named TSO) as well as the goal-oriented technique (called GO) proposed in the current paper is evaluated here in the context of the clustering-adequacy study presented in Section IV. The adequacy results obtained by each studied method (GO or TSO) will be compared with the ones of the reference case. The latter gives us the correct adequacy results without applying the clustering study (i.e., directly relying on the "true" historical hourly FB data). In other words, in the reference case, the exact ("true") hourly FB domain is directly applied to each hourly economic dispatch problem (4)-(9). The closer the results obtained from the clusteringadequacy analyses are to the reference adequacy outcomes, the more efficient and accurate is the clustering approach that has been used. For an effective comparison of results, for a given number of clusters $(k)$, the absolute error (difference) between each zonal adequacy indicator obtained by the clustering-adequacy framework and its reference value is added up and calculated as follows:

$$
\begin{aligned}
\Delta_{L O L P} & =\left|\sum_{i=1}^{N}\left(L O L P_{i}^{t c}-L O L P_{i}^{r e f}\right)\right| \\
\Delta_{T E N S} & =\left|\sum_{i=1}^{N}\left(T E N S_{i}^{t c}-T_{E N S_{i}^{r e f}}\right)\right|
\end{aligned}
$$

where $L O L P_{i}^{t c}$ gives the LOLP in zone $i$ in the test case obtained by either GO or TSO method and $L O L P_{i}^{r e f}$ presents the exact LOLP in zone $i$ in the reference case, where there is no clustering analysis. TENS $S_{i}^{t c}$ and TENS $S_{i}^{\text {ref }}$ denote the TENS in zone $i$ in the test case and the reference case, respectively.

\section{Simulation results}

The performance of studied distance measures (GO and TSO) is evaluated here for various numbers of clusters. Starting with 2 clusters, we conduct the clustering-adequacy simulations using both studied distance measures. Afterwards, we continuously increase the number of clusters $(k)$ to 10 . The adequacy-related indicators are evaluated in each case, and are compared with the reference results, which are obtained by applying the "true" FB domains (without doing any clustering study). It should be noted that the term "true" FB domains do not imply that the FB domains represent the cross-border exchanges with an accurate theoretical formulation since they are obtained upon certain assumptions and simplifications.

Table I presents the errors (according to (10) and (11)) in adequacy results obtained by the clustering-adequacy framework with respect to the correct results of the reference case. In the latter (reference) case, the overall adequacy results i.e., the sum of zonal adequacy indicators equals to $2825 \mathrm{GWh}$ for TENS and $31.8 \%$ for LOLP.

In Table I, it is clear that the clustering methodology of the CWE TSOs leads to the adequacy outcomes, which are not close to the reference results. More importantly, it is observed that the existing differences (errors) between the adequacy results (obtained by the latter approach) and the reference results remain at those high levels for different numbers of clusters $(k)$. As it can be seen, for $k=2$ to 6 , the absolute errors of LOLP stay at around 16\% and the errors relating to TENS vary between 1103 and $1250 \mathrm{GWh}$. Similarly, with $k=7$ to 10 , the errors associating with LOLP and TENS are changing around $14 \%$ and $1000 \mathrm{GWh}$, respectively. This outcome can be explained by the aggregation feature of the distance measure developed by the CWE TSOs, which eventually covers the existing differences of the compared FB domains. Indeed, by analyzing the obtained internal cluster results, knowing 
that the total number of FB domains in the studied dataset is equal to 5808, it is found that there exists always a large cluster consisting of 3239, 3220, 3220, 3217 and 3217 FB domains for $k$ equal to 2 to 6 , respectively. It implies that the FB domains placed in this large cluster are estimated similar to each other according to the employed distance measure. Therefore, those FB domains are assigned to the same cluster for various $k$ while they present different properties in the context of adequacy simulations.

TABLE I. ERRORS IN ADEQUACy RESUlts ObTAINED By the STUDIED DistANCE MEASURES For VARIOUS NUMBERS OF CLUSTER $(K)$

\begin{tabular}{|c|c|c|c|c|c|c|c|c|c|c|}
\hline \multicolumn{2}{|c|}{} & $k=2$ & $k=3$ & $k=4$ & $k=5$ & $k=6$ & $k=7$ & $k=8$ & $k=9$ & $k=10$ \\
\hline \multirow{3}{*}{ TSO } & $\Delta_{\text {LOLP }}(\%)$ & 16.58 & 16.94 & 16.6 & 16.2 & 16.2 & 14 & 14.2 & 13.8 & 13.82 \\
\cline { 2 - 11 } & $\Delta_{T E N S}(\mathrm{GWh})$ & 1250 & 1191 & 1172 & 1105 & 1103 & 973 & 1010 & 995 & 994 \\
\hline \multirow{2}{*}{ GO } & $\Delta_{\text {LOLP }}(\%)$ & 11.6 & 4.37 & 3.75 & 3.11 & 4.04 & 3.03 & 2.94 & 1.98 & 2.44 \\
\cline { 2 - 11 } & $\Delta_{\text {TENS }}(\mathrm{GWh})$ & 1392 & 663 & 609 & 313 & 387 & 199 & 339 & 171 & 180 \\
\hline
\end{tabular}

Regarding the results obtained by the goal-oriented technique shown in Table I, one can observe that the reported errors (with respect to the reference results) are high for $k$ equal to 2 $\left(\Delta_{L O L P}=11.6 \%\right.$ and $\left.\Delta_{T E N S}=1392 \mathrm{GWh}\right)$. This is explained by the fact that having two clusters is not sufficient to form the homogenous clusters. Consequently, the selected cluster prototypes cannot correctly represent their respective FB domains leading to big errors in the adequacy results. However, when the number of clusters increases, it is observed that the errors are generally decreased, and the adequacy results obtained by the goal-oriented technique become closer to the reference results. Indeed, the absolute error of LOLP reduces from $11.6 \%(k=2)$ to $2.44 \%(k=10)$. Similarly, starting from $1392 \mathrm{GWh}(k=2)$, absolute error of TENS decreases to $180 \mathrm{GWh}$ for $k=10$. This feature presents the main advantage of the proposed goal-oriented technique, thanks to which, the accuracy of adequacy assessments in the CWE electricity system can be improved.

By comparing the errors reported in Table I, it can be concluded that the proposed goaloriented clustering outperforms the technique used by the CWE TSOs. The proposed method reduces the existing errors in the adequacy results by a factor of over 5.5 (at $k=10$ as an example), i.e., from $13.82 \%$ to $2.44 \%$ and from 994 to $180 \mathrm{GWh}$. These findings confirm that clustering of FB domains based on their zonal extrema net positions improves the accuracy of adequacy results, and that it can cover the drawbacks of the distance measure being utilized by the CWE TSOs.

\section{DISCUSSION}

On the accuracy of studied distance measures: The simulations and analyses conducted in this paper demonstrate that the clustering of FB domains based on the proposed goal-oriented technique leads to the adequacy results, which are noticeably more accurate than the ones obtained according to the clustering approach used by the TSOs. The inaccurate estimation of cross-border exchange capacities in the latter approach is due to consideration of all the vertices of FB domain and aggregation of all the individual distances between the vertices of 
two FB domains. By aggregating those individual distances, the properties relating to the vertices that are decisive in adequacy assessments are lost (in the final aggregated value between two FB domains). In addition, the FB domains present in dataset are considered rather similar to each other; hence, a majority of them will be assigned to the same cluster, while they have different properties in the context of adequacy assessments.

On the time complexity of studied distance measures: The proposed goal-oriented technique considerably reduces the calculation burden of the clustering procedure as it only considers the extrema (maximal/minimal) zonal net positions of each FB domain to construct the distance matrix that includes the pairwise dissimilarities of FB domains in studied dataset. To illustrate it, we conduct a numerical exercise to calculate the pairwise distances between 100 FB domains. The goal-oriented distance measure requires $2.1 \mathrm{~s}$ to perform such an operation while the distance measure used by the CWE TSOs needs $240 \mathrm{~s}$ to conduct the same analysis. It is worth noting that this is the time needed for constructing a $100 \times 100$ matrix (comparison of $100 \mathrm{FB}$ domains). For a larger dataset, the required time will accordingly increase.

On the scalability issue of distance measure employed by the CWE TSOs: The number of vertices of a FB domain polytope is highly sensitive to the number of its dimensions. For instance, 140 linear constraints representing a typical FB domain has 52 vertices in 4D, 787 vertices in 5D, and 2764 vertices in $6 \mathrm{D}$ space. Due to consideration of all the vertices of FB domain as well as the aggregation principle of the distance measure employed by the CWE TSOs, it is expected that this approach would face further problems in terms of accuracy and time complexity when the number of countries involved in the FBMC increases. According to the evolution roadmap of the FBMC project [31], it is planned that by February 2022, the FB approach will be extended to 13 countries (the core group countries) in the framework of the Single Day-Ahead Coupling (SDAC) in Europe.

On the proper number of clusters: The required number of clusters should be defined in accordance with the characteristics of our dataset i.e., the number of historical FB domains and their variability in terms of forms and shapes. Although increasing the number of clusters may lead to less intra cluster variation, hence, higher quality cluster prototypes, the effective solution will not be to choose a great number of clusters since it complicates the correlation analysis to be done after the clustering study (to link the cluster results to the exogenous factors of the FBMC). Consequently, to define the optimal number of clusters, it is needed to evaluate the overall error in the whole clustering-correlation framework. It should be noted that identifying the optimal number of clusters when the distance measure of TSOs is employed in adequacy assessments is of a minor relevance since the improvement of accuracy with increasing the number of clusters is almost negligible.

In order to summarize the above-mentioned points, Table II presents a brief comparison of the proposed goal-oriented clustering of the FB domains with the approach being used by the TSOs, in the context of adequacy assessments of the CWE electricity system. 
TABLE II. COMPARATIVE ANALYSIS OF THE GOAL-ORIENTED CluSTERING TECHNIQUE AND THE APPROACH USED BY THE TSOS IN THE CONTEXT OF ADEQUACY STUDY

\begin{tabular}{|c|l|c|c|}
\hline \multicolumn{2}{|c|}{} & TSO & GO \\
\hline $\mathbf{1}$ & $\begin{array}{l}\text { Accuracy in the FB domain } \\
\text { assignment task }\end{array}$ & Not accurate & $\begin{array}{c}\text { Over 5.5 times more accurate } \\
\text { (e.g., with } k=10)\end{array}$ \\
\hline $\mathbf{2}$ & Calculation time & Computationally heavy & Over 100 times faster \\
\hline $\mathbf{3}$ & $\begin{array}{l}\text { Scalability with the future } \\
\text { evolution of the FBMC }\end{array}$ & $\begin{array}{c}\text { Not compatible, considering } \\
\text { all the vertices of FB domains }\end{array}$ & $\begin{array}{c}\text { Compatible, focusing on the } \\
\text { specific important vertices }\end{array}$ \\
\hline $\mathbf{I m p a c t ~ o f ~ t h e ~ n u m b e r ~ o f ~}$ & $\begin{array}{c}\text { No significant improvement } \\
\text { found by changing } k\end{array}$ & $\begin{array}{c}\text { Noticeable improvements found } \\
\text { by increasing } k \text {, a trade-off } \\
\text { between } k \text { and the gained } \\
\text { accuracy should be obtained }\end{array}$ \\
\hline
\end{tabular}

\section{CONCLUSION}

This paper presents a novel data-driven approach for enhancing integration of cross-border exchange capacities through the FB domains into the adequacy assessments of $\mathrm{CWE}$ electricity system. To this end, we leverage the historical FB data to determine the FB domain that can properly match with each generated scenario of the probabilistic adequacy assessments. The goal-oriented clustering of historical FB domains is proposed here according to which, the FB domains are grouped based on their zonal extrema net positions. This choice is motivated by the fact that at the time of shortage, which shapes the adequacy assessment outcomes, the zonal exchanges tend to reach their extrema capacities allowed by the FB domain to minimize the costs of energy not supplied. The performance of the proposed goaloriented clustering technique is examined within the developed clustering-adequacy framework. The simulation results reveal that the proposed technique can improve the accuracy of the FB domain assignment task. The main findings of this paper underpin that in an adequacy assessment context, the overall shape of the FB domain is not important, instead, the vertices representing the maximum cross-zonal exchanges are the decisive factors. In addition, it is found that relying on all the vertices of FB domain, which is the current approach being used by the TSOs, can cause further problems in terms of computational time and scalability when the new countries join to the FBMC. The salient features of proposed clustering technique with respect to the approach currently employed by the TSOs are thus its enhanced accuracy, scalability with the evolution of the FBMC, and required computation time. It is worth noting that any clustering technique for FB domains to be utilized in the Monte Carlo-based adequacy assessments should be complemented with a correlation or classification study that links the clustered FB domains to the explanatory variables affecting them. This latter topic constitutes our future research direction.

\section{FUNDING}

This research was carried out in the context of the Energy Transition Fund, ADABEL project, supported by the FPS Economy, S.M.E.s, Self-Employed and Energy, Belgium. 
NOMENCLATURE

Acronyms:

$\begin{array}{ll}\text { CWE } & \text { Central Western Europe } \\ \text { D2CF } & \text { 2 Days-Ahead Congestion Forecast } \\ \text { ENS } & \text { Energy Not Served } \\ \text { FB } & \text { Flow-Based } \\ \text { FBMC } & \text { Flow-Based Market Coupling } \\ \text { GO } & \text { Goal-Oriented } \\ \text { NTC } & \text { Net Transfer Capacity } \\ \text { PAM } & \text { Partitioning Around Medoids } \\ \text { PTDF } & \text { Power Transfer Distribution Factors } \\ \text { RAM } & \text { Remaining Available Margin } \\ \text { SDAC } & \text { Single Day-Ahead Coupling } \\ \text { TENS } & \text { Total Energy Not Served } \\ \text { TSOs } & \text { Transmission System Operators } \\ \text { 2D } & \text { Two-dimensional } \\ \text { 5D } & \text { Five-dimensional }\end{array}$

Indices:

$t$

Hourly time step in adequacy simulations

$i$

Index of zone in the interconnected CWE electricity system

Parameters:

$\begin{array}{ll}\boldsymbol{A} & \text { Inequality matrix in a system of linear constraints } \\ \boldsymbol{b} & \text { The right-hand side vector in a system of linear constraints } \\ N P_{A, i}^{\max } & \text { Maximum net position of zone } i \text { defined by the FB domain A } \\ N P_{A, i}^{\min } & \text { Minimum net position of zone } i \text { defined by the FB domain A } \\ d_{G O}\left(F B_{A}, F B_{B}\right) & \text { Distance (dissimilarity) between FB domains A and B according to the } \\ & \text { goal-oriented dissimilarity measure } \\ d_{T S O}\left(F B_{A}, F B_{B}\right) & \begin{array}{l}\text { Distance (dissimilarity) between FB domains A and B according to the } \\ \text { dissimilarity measure employed by the TSOs }\end{array} \\ N & \text { Number of zones in the interconnected CWE electricity system } \\ L D_{i} & \text { Load demand in zone } i \\ N P_{i} & \text { Net position of zone } i \\ G_{i}^{\text {max }} & \text { Maximum available generation in zone } i \\ \boldsymbol{P T D F} & \text { Power transfer distribution factor matrix } \\ \boldsymbol{N P} & \text { Vector of zonal net positions } \\ \boldsymbol{R A M} & \text { Vector of remaining available margin of selected elements }\end{array}$


$\Delta_{L O L P} \quad$ LOLP error between the true result (no clustering) and the clusteringadequacy result

$\Delta_{\text {TENS }} \quad$ TENS error between the true result (no clustering) and the clusteringadequacy result

LOLP $P_{i}^{t c} \quad$ LOLP index in zone $i$ in the test case (obtained by either the GO or TSO method)

$L O L P_{i}^{r e f} \quad$ LOLP index in zone $i$ in the reference case (no clustering)

TENS $S_{i}^{t c}$

TENS in zone $i$ in the test case (obtained by either the GO or TSO method)

TENS $_{i}^{\text {ref }} \quad$ TENS in zone $i$ in the reference case (no clustering)

Variables:

$\boldsymbol{x}$

Vector of decision variables in the system of linear constraints

$E N S_{i}$

Energy not served in zone $i$

$G_{i}$

Generation in zone $i$

\section{REFERENCES}

[1] J-F Toubeau, J. Bottieau, F. Vallée, Z. De Grève, "Deep learning-based multivariate probabilistic forecasting for short-term scheduling in power markets," IEEE Transactions on Power Systems. vol. 34, no. 2, pp. 1203-1215, 2018.

[2] J-F. Toubeau, B. Bakhshideh Zad, M. Hupez, Z. De Grève, F. Vallée, "Deep reinforcement learning-based voltage control to deal with model uncertainties in distribution networks," Energies, vol. 13, no. 15, 2020.

[3] J. Wang, A. Botterud, R. Bessab, H. Kekob, et. al. "Wind power forecasting uncertainty and unit commitment', Applied Energy, vol. 88, no. 11, pp. 4014-4023, 2011.

[4] J. Wei, Y. Zhang, J. Wang, X. Cao, M. Armoghan Khan, "Multi-period planning of multienergy microgrid with multi-type uncertainties using chance constrained information gap decision method," Applied Energy, vol. 260, 114188, 2020.

[5] "Identification of appropriate generation and system adequacy standards for the internal electricity market," European commission, 2016.

[6] R. Billinton, L. Wenyuan, "Reliability assessment of electric power systems using Monte Carlo methods," Springer, Boston, MA, 1994.

[7] F. Vallée, G. Brunieau, M. Pirlot, O. Deblecker, J. Lobry, "Optimal wind clustering methodology for adequacy evaluation in system generation studies using nonsequential Monte Carlo simulation," IEEE Transactions on Power Systems, vol. 26, no. 4, pp. 21732184, 2011.

[8] R. Billinton, R. Karki, Y. Gao, D. Huang, P. Hu, W. Wangdee, "Adequacy assessment considerations in wind integrated power systems," IEEE Transactions on Power Systems, vol. 27, no. 4, pp. 2297-2305, 2012.

[9] E. Tómasson, L. Söder, "Generation adequacy analysis of multi-area power systems with a high share of wind power," IEEE Transactions on Power Systems, vol. 33, no. 4, pp. 3854-3862, 2018. 
[10] A. Salehi Dobakhshari, M. Fotuhi-Firuzabad, "A reliability model of large wind farms for power system adequacy studies," IEEE Transactions on Energy Conversion, vol. 24, no. 3, pp. 792-801, 2009.

[11] S. Sulaeman, M. Benidris, J. Mitra, C. Singh, "A wind farm reliability model considering both wind variability and turbine forced outages," IEEE Transactions on Sustainable Energy, vol. 8, no. 2, pp. 629-637, 2017.

[12] X. Zhuang, C. Ye, Y. Ding, L. Cheng, Y. Song, S. Ye, S. Tian, R. Chen, "Data-driven efficient reliability evaluation of power systems with wind penetration: an integrated GANs and CE method," IET Generation, Transmission \& Distribution, vol. 14, no. 4, pp. 577-584, 2020.

[13] N. Amjadi, "Generation adequacy assessment of power systems by time series and fuzzy neural network," IEEE Transactions on Power Systems, vol. 21, no. 3, pp. 13401349, 2006.

[14] M. Cepeda, M. Saguan, D. Fino, V. Pignon, "Generation adequacy and transmission interconnection in regional electricity markets," Energy Policy, vol. 37, pp. 5612-5622, 2009.

[15] "Adequacy and flexibility study for Belgium 2020-2030," Elia, 2019, available at: https://www.elia.be/en/electricity-market-and-system/adequacy/adequacy-studies

[16] 'Modelling of flow-based domains in Antares for adequacy studies', RTE, 2017, available at: https://antares-simulator.org/media/files/page/ZHX0N-171024-RteModelling-of-Flow-Based-Domains-in-Antares-for-Adequacy-Studies.pdf

[17] D. Schönheit, R. Weinhold, C. Dierstein, "The impact of different strategies for generation shift keys (GSKs) on the flow-based market coupling domain: A model-based analysis of Central Western Europe," Applied Energy, Vol. 258, 114067, 2020.

[18] P. Henneaux, P. Lamprinakos, G. de Maere d'Aertrycke, K. Karoui, "Impact assessment of a minimum threshold on cross-zonal capacity in a flow-based market, Electric Power Systems Research," 190, 106693, 2021.

[19] K. Van den Bergh, E. Delarue, "An improved method to calculate injection shift keys,” Electric Power Systems Research, vol. 134, pp. 197-204, 2016.

[20] D. Schönheit, M. Kenis, L. Lorenz, D. Möost, E. Delarue and K. Bruninx, "Toward understanding flow-based market coupling: An open-access model," Advances in Applied Energy, vol. 2, 100027, 2021.

[21] "Mid-term adequacy forecast, ENTSO-E," 2019, available at: https://eepublicdownloads.entsoe.eu/clean-documents/sdcdocuments/MAF/2019/MAF\%202019\%20Appendix\%202\%20-\%20Methodology.pdf

[22] "Typical flow-based days selection," RTE, 2017, available at: https://antaressimulator.org/media/files/page/7NY5W-171024-Rte-Typical-Flow-Based-DaysSelection-1.pdf

[23] "Bilan prévisionnel de l'équilibre offre-demande d'électricité en France," RTE, 2019, avalable at: https://assets.rte-france.com/prod/public/202006/bilan\%20pr\%C3\%A9visionnel\%202019\%20rapport $\% 20$ technique compressed.pdf .

[24] "Adequacy study for Belgium: the need for strategic reserve for winter 2020-21," Elia, 2019, available at: https://www.elia.be/-/media/project/elia/shared/documents/eliasite/studies/2019/strategic-reserve-for-winter-2020-21.pdf?la=en 
[25] B. Bakhshideh Zad, B. Vatandoust, J-F. Toubeau, Z. De Grève, F. Vallée, “Advanced clustering of flow-based domains for adequacy study purposes" the $14^{\text {th }}$ IEEE PowerTech conference, 2021, Madrid, Spain.

[26] K. Van den Bergh, J. Boury, E. Delarue, "The flow-based market coupling in central western Europe: concepts and definitions," The Electricity Journal, vol. 29, no. 1, pp. 2429, 2016.

[27] Joint allocation office, Market data, Implicit allocation, available at: https://www.jao.eu/marketdata/implicitallocation

[28] D. Avis. "A revised implementation of the reverse search vertex enumeration algorithm, in Polytopes - Combinatorics and Computation," Springer, pp. 177-198, 2000.

[29] L. Kaufman. P. J. Rousseeuw. "Finding groups in data: an introduction to cluster analysis," vol. 39, Wiley Online Library, 1990.

[30] E. Heylen, G. Deconinck, D.Van Hertem, "Review and classification of reliability indicators for power systems with a high share of renewable energy sources," Renewable and Sustainable Energy Reviews, vol. 97, pp. 554-568, 2018.

[31] Joint allocation office, Core FBMC project, https://www.jao.eu/core-fb-mc 